\title{
The complex structure of hunter-gatherer social networks
}

\author{
Marcus J. Hamilton ${ }^{1, *}$, Bruce T. Milne ${ }^{2}$, Robert S. Walker ${ }^{3}$, \\ Oskar Burger ${ }^{1}$ and James H. Brown ${ }^{2,4}$
}

\author{
${ }^{1}$ Department of Anthropology, and ${ }^{2}$ Department of Biology, University of New Mexico, Albuquerque, NM 87131, USA \\ ${ }^{3}$ Department of Anthropology, University of Colorado at Denver and Health Sciences Center, Denver, CO 80217, USA \\ ${ }^{4}$ Santa Fe Institute, 1399 Hyde Park Road, Santa Fe, NM 87501, USA
}

\begin{abstract}
In nature, many different types of complex system form hierarchical, self-similar or fractal-like structures that have evolved to maximize internal efficiency. In this paper, we ask whether hunter-gatherer societies show similar structural properties. We use fractal network theory to analyse the statistical structure of 1189 social groups in 339 hunter-gatherer societies from a published compilation of ethnographies. We show that population structure is indeed self-similar or fractal-like with the number of individuals or groups belonging to each successively higher level of organization exhibiting a constant ratio close to 4 . Further, despite the wide ecological, cultural and historical diversity of hunter-gatherer societies, this remarkable self-similarity holds both within and across cultures and continents. We show that the branching ratio is related to density-dependent reproduction in complex environments and hypothesize that the general pattern of hierarchical organization reflects the self-similar properties of the networks and the underlying cohesive and disruptive forces that govern the flow of material resources, genes and non-genetic information within and between social groups. Our results offer insight into the energetics of human sociality and suggest that human social networks self-organize in response to similar optimization principles found behind the formation of many complex systems in nature.
\end{abstract}

Keywords: hunter-gatherers; social networks; self-similarity; generalized Horton analysis; population structure; complex systems

\section{INTRODUCTION}

Hunter-gatherer societies are embedded within complex ecosystems, systems that are organized at multiple scales by the fluxes and exchanges of energy and matter between organisms and their environment (Schneider \& Kay 1994; Brown et al. 2004). Hunter-gatherers harvest resources from these ecosystems to meet basic metabolic and material requirements by adjusting group size and organization in response to the spatial and temporal variation in resource distribution. Recently, we showed that within huntergatherer societies, the space required by an individual to meet metabolic demand decreases in larger populations, introducing an important economy of scale where population level energetic efficiency increases with population size (Hamilton et al. 2007). We hypothesized that this scaling relation results from the complex structure of underlying social networks, which serve to redistribute heterogeneously distributed fitness-related resources, such as energy, materials and information, within the environment to group members (Hamilton et al. 2007). In this paper, we investigate this hypothesis further by analysing the structural organization of a large sample of ethnographic huntergatherer societies.

In an innovative study, Zhou et al. (2005) showed that human social groups form a hierarchy of discrete group

\footnotetext{
* Author for correspondence (marcusj@unm.edu).
}

Electronic supplementary material is available at http://dx.doi.org/10 1098/rspb.2007.0564 or via http://www.journals.royalsoc.ac.uk. sizes with a constant scaling ratio of approximately 3 . Here, we ask three questions: (i) do we find similar hierarchical scaling relations within hunter-gatherer societies? (ii) If so, how do these scaling relations vary across societies? and (iii) what mechanisms might be hypothesized for such scaling relations?

Hierarchical networks are common in nature. Many exhibit self-similar scaling properties that reflect fundamental physical, chemical and biological constraints on their structure and dynamics (Rodriguez-Iturbe \& Rinaldo 1997; Brown et al. 2002; Ravasz \& Barabási 2003; Colizza et al. 2004; Sole \& Bascompte 2006). Complex systems composed of multiple interacting parts tend to self-organize or evolve structures that maximize whole-system performance by optimizing the interactions among components (Colizza et al. 2004; Rinaldo et al. 2006). In hierarchical systems, it has been shown theoretically and empirically that these optimal networks are self-similar and fractal-like (West et al. 1997, 1999; Ravasz \& Barabási 2003; West \& Brown 2005; Rinaldo et al. 2006). Such networks optimize the distribution of material or energy by minimizing network size and resistance (Banavar et al. 1999, 2002; West \& Brown 2005; Rinaldo et al. 2006). Self-similar or fractal networks are characterized quantitatively by constant ratios across successive levels and power-law distributions (RodriguezIturbe \& Rinaldo 1997; West et al. 1997, 1999). True mathematical fractals continue indefinitely, whereas real physical, biological and human systems are often described as fractal-like because they are finite, asymptotic 
and truncated, with the sizes of both the terminal units and the total network being constrained. Empirical examples of such networks range from the branching tributaries in river drainages (Rodriguez-Iturbe \& Rinaldo 1997) to the vascular systems that distribute energy and materials within the bodies of animals and plants (West et al. 1997; Enquist et al. 2000). Analogously, selfsimilarity in human societies may have evolved to optimize the acquisition and distribution of fitness-related resources to group members.

Many contemporary human social systems form complex social networks where individuals are connected to each other at multiple levels of organization. Examples include the structure of e-mail networks (Guimera et al. 2003), the formation of cliques in the US House of Representatives (Porter et al. 2005), networks of jazz musicians (Arenas et al. 2004), actors (Newman 2003) and co-authors of scientific publications (Newman 2004). We suggest that hunter-gatherer social organization can also be viewed as a social network. The network arises from interactions and exchanges of energy, material and information between individuals, which occur within the context of a hierarchical group structure. This hierarchical structure is constrained externally by seasonal variation in local ecological conditions and internally by the human life history. In foraging societies, energy and material flows typically include the exchange of food resources, trade goods and raw materials for tools, clothing and shelter, and information transfers include both gene flow through reproduction and the exchanges of many kinds of culturally transmitted information by means of language or other signals.

Traditional hunter-gatherer societies exhibit hierarchical structures (Birdsell 1958, 1993; Kelly 1995; Gamble 1999; Binford 2001; Maschner \& Bentley 2003), in which individuals form a nested series of discrete, yet flexible social units that occupy space and exchange energetic, material and informational resources at differential rates (Johnson 1982). Individuals are nested within nuclear families, formed to provide the parental investment required to rear dependent offspring (Kaplan et al. 2000; Gurven \& Walker 2006). Families fission and fuse to form larger residential foraging groups, which tend to increase the rate and decrease the variance of resource acquisition (Gurven 2004), and which change in size and composition in response to temporal and spatial changes in the environment (Kelly 1995). These extended families are members of still larger groups that are dispersed over larger areas and interact with decreasing frequency, but serve to maintain social ties, conduct trade and information exchange, perform ceremonies and exchange marriage partners (Stewart 1938; Gould 1969; Lourandos 1997; Binford 2001).

Considerations of group size and social organization in traditional human societies commonly emphasize group foraging (Kelly 1995), cognitive capacity (Dunbar 1993), demographic variance (Wobst 1974) and the various mechanisms such as group fissioning, mass rituals, and political hierarchies (Maschner \& Bentley 2003) that have evolved as a consequence of the cohesion and tension inherent to living in large populations (Johnson 1982). While all these mechanisms must play important roles individually, of interest here is whether hunter-gatherer social organizations as a whole form self-similar structures.
If so, this would suggest that hunter-gatherer social structures may have self-organized to optimize energy, material and information flows among group members.

\section{DATA}

We used a recent compilation of ethnographic data that contains estimates of group sizes at multiple levels of organization (Binford 2001). This dataset is a global sample of 339 hunter-gatherer societies, representing diverse foraging strategies, inhabiting a wide range of ecological conditions, and including multiple genetic, racial and ethnic groups. The dataset includes 1189 estimates of group size, from family units and seasonal residential groups, to periodic aggregations and regional populations. A sample size this large is rare in cross-cultural studies and allows for powerful quantitative analyses. The original data were obtained from published ethnographies (Binford 2001), which undoubtedly varied in methodology. However, if we can assume that the estimates of group sizes within each of these ethnographies are independent and unbiased because they were obtained by different original investigators, then errors in these estimates will also be independent and unbiased, allowing underlying statistical trends to be recovered despite variation due to methodology and other uncontrolled and unreported factors.

We analysed this dataset using generalized Horton laws, a statistical approach designed to characterize the quantitative structure of hierarchical branching networks (Rodriguez-Iturbe \& Rinaldo 1997; Peckham \& Gupta 1999; Veitzer \& Gupta 2000). Hunter-gatherer group sizes, $g$, can be assigned to hierarchical organizational levels (Johnson 1982; Kelly 1995; Binford 2001). In Horton analysis, these levels are termed Horton orders, $\omega$, from the first-order terminal units to the highest order, $\Omega$. We followed Binford (2001) in recognizing six levels defined as follows: $g_{1}$, single individuals; $g_{2}$, families estimated by dividing total population size by the number of married males, a common technique for estimating family size in the absence of specific demographic data; $g_{3}$, dispersed extended family groups defined as the average size of residential groups during the most dispersed phases of the mobility cycle; $g_{4}$, aggregated groups defined as the average size of residential groups during the most aggregated phases of the mobility cycle; $g_{5}$, periodic aggregations defined as multi-group socio-economic aggregations occurring at periods usually greater than every year; and $g_{\Omega}$, regional populations defined as the total size of regional ethnic units (definitions taken from Binford (2001)). Importantly, Binford defined these groups a priori, so that if a specific estimate for a particular group size was not available from an ethnography, no value was recorded. In addition, it is important to note that while these levels are necessarily hierarchical, there is considerable overlap between group sizes at each level, particularly above the level of families (figure 1). Further, a higher organizational level does not necessarily imply more individuals (so, for example, regional periodic aggregations may include multiple regional populations). We discuss these points further below.

\section{MATERIAL AND METHODS}

To characterize the scaling within populations, we first consider the relation between group sizes across hierarchical 


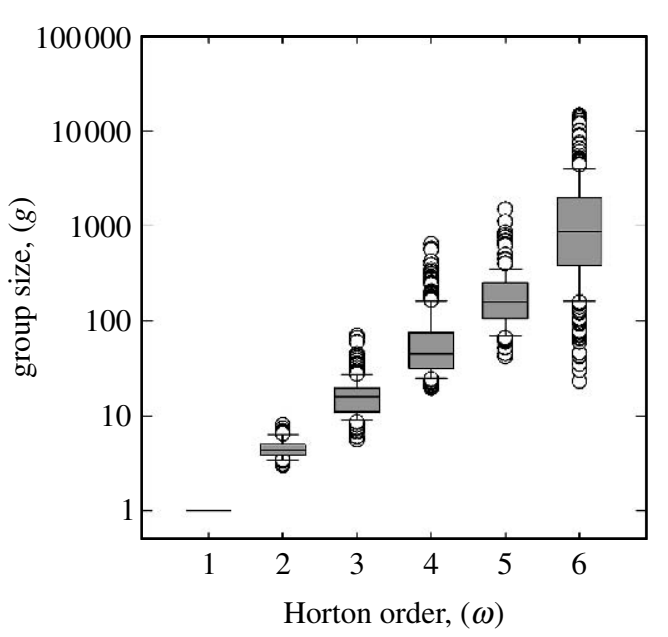

Figure 1. Boxplots of hunter-gatherer group sizes, $g$, as a function of Horton order, $\omega$. The grey boxes encompass $\pm 67 \%$ CIs, the single horizontal lines within the boxes are medians, the paired horizontal lines outside the boxes encompass the $95 \%$ CIs and open circles are outliers (outside the $95 \%$ CIs). All distributions are approximately lognormal. Note the considerable overlap in all group sizes.

orders. Let $g_{i}^{(\omega)}$ be a group of size $g$ in the $i$ th population at order $\omega$. To calculate the average branching ratio, $B$, between the six orders across all populations, we first calculate the number of groups of size $g$ at order $\omega$ within a population. Let $N\left(g_{i}^{(\omega)}\right)=g_{i}^{(\Omega)} / g_{i}^{(\omega)}$ be the number of groups $g$ of order $\omega$ within the $i$ th population. The Horton-Strahler branching ratio is then defined as

$B=\frac{N\left(g_{i}^{(\omega-1)}\right)}{N\left(g_{i}^{(\omega)}\right)}$.

The network structure is self-similar if the branching ratio, $B$, is constant between all levels. Rearranging equation (3.1), on the log scale we have

$\ln N\left(g_{i}^{(\omega+k)}\right)=\ln N\left(g_{i}^{(\omega)}\right)+\lambda_{i} k$

where $\lambda=\ln B$ and $k=\Delta \omega$. Hence, if the network is selfsimilar, then a semi-log plot of $\ln N\left(g_{i}^{(\omega)}\right)$ by $\omega$ will be fit by a straight line with the slope $\lambda$, giving an estimate of the branching ratio, $B$. We first calculated the branching ratio of the mean number of groups per population, $B=\bar{N}\left(g_{i}^{(\omega-1)}\right) /$ $\bar{N}\left(g_{i}^{(\omega)}\right)$ to establish whether they are self-similar across the five branchings (where $\bar{x}$ denotes the geometric mean). To calculate a more accurate estimate of the average branching ratio across populations, we fit equation (3.2) to each population in turn and took the mean branching ratio across populations. We then decomposed the populations by continent and analysed the data similarly to look at variation across continents.

Second, to examine the variation in the branching ratio across the 339 societies, we used generalized Horton analysis, which considers the scaling of not only the means but the entire distributions of groups at each hierarchical level (Veitzer \& Gupta 2000). Defining $p\left(N\left(g_{\omega}\right)\right)$ as the probability distribution of the number of groups $g$ at order $\omega$ per population, if the network is self-similar for all values of $B$, the entire distributions at each successive order can be rescaled by a constant coefficient. Hence, rescaling each probability distribution $p\left(N\left(g_{\omega}\right)\right)$ by its respective mean $\left\langle N\left(g_{\omega}\right)\right\rangle$ non-dimensionalizes the data (where $\langle\cdot\rangle$ denotes the arithmetic mean) and, provided that the distributions are indeed self-similar, collapses all of them onto a single scaling function. Since this form of rescaling does not assume normality and requires only that the mean is finite, we rescaled each value of the number of groups within a population by dividing by the arithmetic mean to give $r_{i}^{(\omega)}=$ $N\left(g_{i}^{(\omega)}\right) /\left\langle N\left(g_{i}^{(\omega)}\right)\right\rangle$ and plotted the resulting distributions. We supplement these analyses with sensitivity analyses in the electronic supplementary material.

\section{RESULTS}

\section{(a) Overall branching ratio}

Mean group sizes, variances and numbers of groups per population are shown in figure 1 and table 1 . The geometric mean of group sizes varied from 4.5 individuals per family to populations of about 840 individuals. Figure 2 shows the semi-log plot of average number of groups per order by order for both the entire dataset and by continent. All plots are well fit by a linear function, as all data points are encompassed by the $95 \%$ confidence limits around the line. This means that the frequency of groups at each level of these societies is statistically selfsimilar and this self-similarity holds not only for the entire dataset, but also individually within each continent. Table 1 provides more detailed results. The slope of the function for the entire dataset is $\lambda=1.28$, estimating the branching ratio between order means at $B=3.60$ (3.23-4.02, 95\% bootstrapped confidence limits). However, this estimate is potentially biased by taking averages for each order, so a better estimate is shown in figure 3, the distribution of the mean branching ratios across all 339 societies, where the overall geometric mean $\bar{B}=3.77$ (3.68-3.87).

\section{(b) Branching ratios by continent}

Figure 2 shows that the branching ratios within each continent are also self-similar as the distributions are well fit by a linear function. However, the slopes between continents vary from $\lambda=1.19-1.33$, reflecting continental differences in the mean branching ratios within the sampled populations. Table 2 gives exact estimates of the mean branching ratios, which vary from $B=3.37-$ 3.95. Table 2 also gives mean family size, $F$, estimates by continent, which range from $F=3.89-6.25$. The Australian mean family size of 6.25 is probably affected by high levels of polygyny in traditional aboriginal societies (Binford 2001).

\section{(c) Rescaling}

Figure 4 shows the results of the data collapse. The data fall onto a single curve showing the remarkable selfsimilarity of group sizes at each level across all societies. The data collapse demonstrates not only that mean group size per order scales nearly identically across orders, but also the entire form of the distribution at each order exhibits the same scaling across orders and across societies. Hence, not only are individual societies structurally self-similar, but also that this self-similarity holds across all 339 societies and all branching ratios.

\section{DISCUSSION}

Our results show that hunter-gatherer societies throughout the world exhibit remarkably similar hierarchical organizations. These societies self-organize into hierarchical self-similar networks of predictable group sizes that 
Table 1. Hunter-gatherer group sizes and frequencies.

\begin{tabular}{|c|c|c|c|c|c|c|c|}
\hline \multirow{2}{*}{$\begin{array}{l}\text { organizational level } \\
\text { group size }(g)\end{array}$} & $\begin{array}{l}\text { Horton } \\
\text { order }\end{array}$ & sample size & ln mean & s.d. & $\begin{array}{l}\text { geometric } \\
\text { mean }\end{array}$ & $95 \% \mathrm{CLs}^{1}$ & $95 \% \mathrm{CLs}$ \\
\hline & $\omega$ & $n$ & $\langle\ln g\rangle$ & $s_{g}$ & $\bar{g}$ & lower & upper \\
\hline individual & 1 & - & 0 & - & 1 & - & - \\
\hline family & 2 & 114 & 1.50 & 0.23 & 4.48 & 4.31 & 4.67 \\
\hline dispersed group & 3 & 227 & 2.75 & 0.46 & 15.60 & 14.68 & 16.58 \\
\hline aggregated group & 4 & 297 & 3.98 & 0.71 & 53.66 & 49.86 & 58.29 \\
\hline periodic aggregation & 5 & 213 & 5.11 & 0.66 & 165.32 & 152.25 & 181.00 \\
\hline population size & 6 & 339 & 6.73 & 1.25 & 839.19 & 736.36 & 954.03 \\
\hline frequency, $N(g)$ & $\omega$ & $n$ & $\langle\ln N(g)\rangle$ & $s_{N(g)}$ & $\bar{N}(g)$ & lower & upper \\
\hline individual & 1 & 339 & 6.73 & 1.25 & 839.19 & 725.72 & 949.31 \\
\hline family & 2 & 213 & 4.91 & 1.20 & 136.29 & 108.63 & 169.81 \\
\hline dispersed group & 3 & 297 & 3.78 & 1.15 & 43.60 & 37.50 & 50.36 \\
\hline aggregated group & 4 & 227 & 2.72 & 1.14 & 15.25 & 13.39 & 17.39 \\
\hline periodic aggregation & 5 & 114 & 1.53 & 1.06 & 4.63 & 4.01 & 5.27 \\
\hline population & 6 & - & 0 & - & 1 & - & - \\
\hline
\end{tabular}

${ }^{a}$ Ninety-five per cent bootstrapped confidence limits.

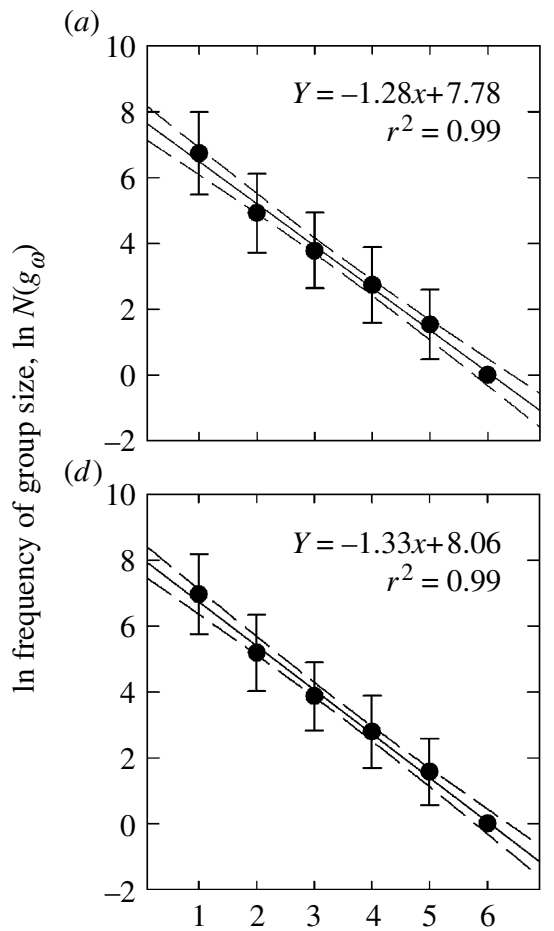

(b)

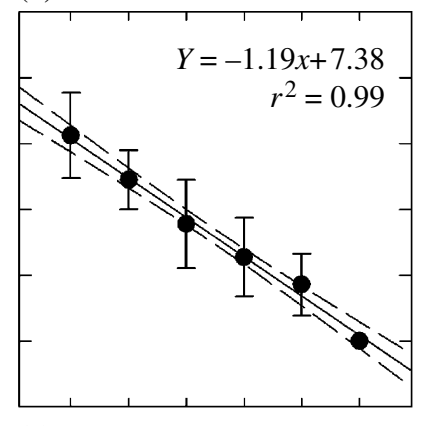

$(e)$

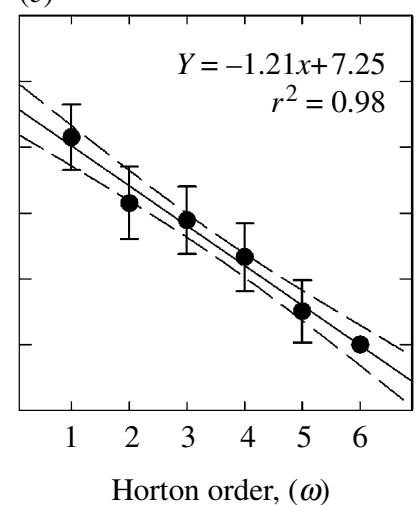

$(c)$

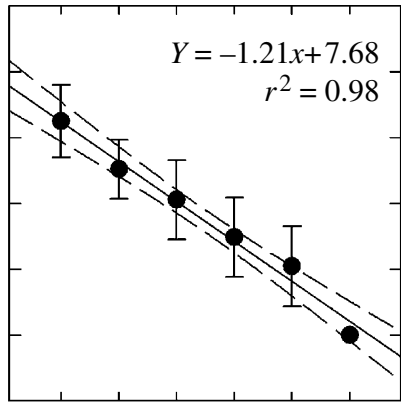

$(f)$

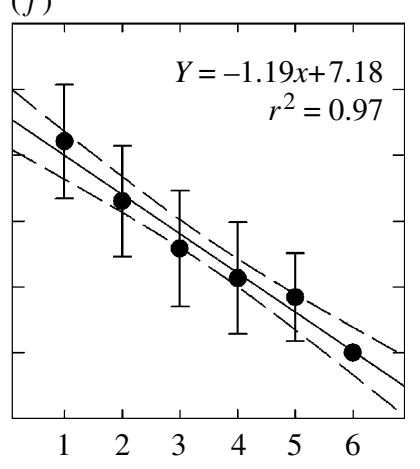

Figure 2. Horton plots of mean number of groups per population for the entire data set and the data decomposed by continent. (a) All data, $n=339$; (b) Asia, $n=29$; (c) Africa, $n=19$; (d) North America, $n=216$; (e) Australia, $n=56$; $(f)$ South America, $n=19$. All the distributions are well fit by linear functions as the $95 \%$ CIs around the slopes encompass all data points in all cases (error bars are 1 s.d.). Slopes vary from 1.19 to 1.33 reflecting variation in branching ratios across continents (table 2 and $\S 5$ for further details).

scale at a constant rate across all successive levels. Our analyses show that not only are these societies internally self-similar, but also that this self-similarity is found across societies that differ widely in ecological, historical and genetic backgrounds. However, there is variation in the branching ratios across continents and we hypothesize about possible reasons for this below. One hypothesis that might conceivably explain the documented self-similarity in general is that it is an artefact, reflecting either how ethnographers collected and interpreted the original data, or how Binford compiled and coded their data. The latter cannot be true, because the group levels were defined $a$ priori (e.g. prior to the coding process: see \$3). The considerable overlap between successive group sizes shown in figure 1 and table 1 suggests that the reported group sizes at different levels are, indeed, independent estimates. Although we cannot completely exclude the possibility that ethnographers are inherently disposed to categorize hunter-gatherer social systems into hierarchically structured groups exhibiting self-similar scaling 
(a)

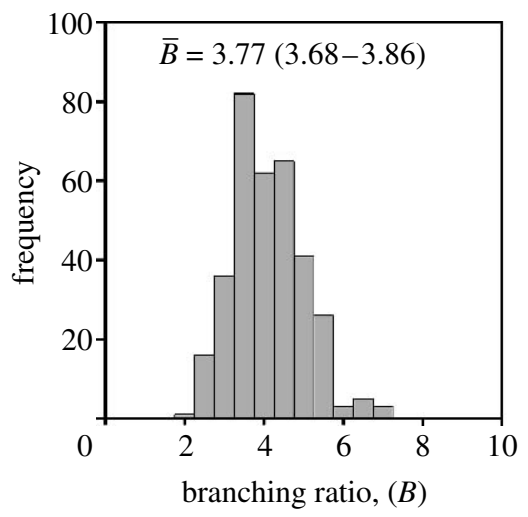

(b)

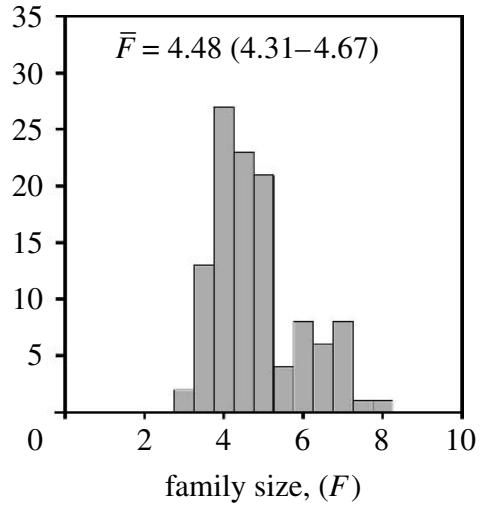

Figure 3. Distributions of (a) branching ratios $(n=339)$ and $(b)$ family sizes $(n=114)$ over the entire data set. Both distributions are approximately lognormal and average values given are geometric means with $95 \%$ bootstrap confidence limits. Values for the data decomposed by continent are given in table 2 .

(a)

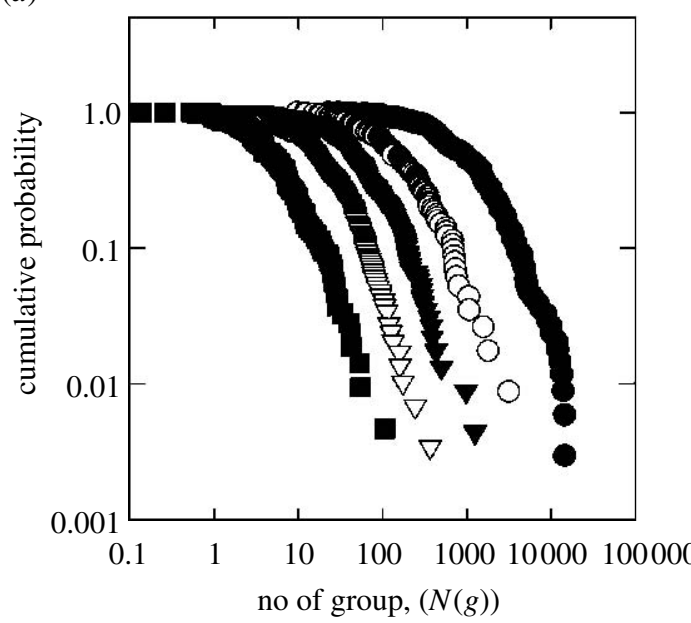

(b)

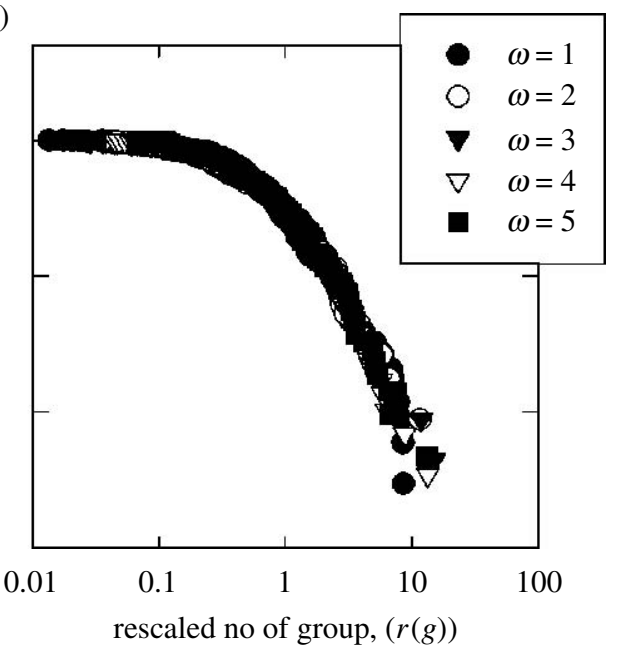

Figure 4. Data collapse of cumulative probability distributions. (a) Probability distributions for the frequency of group sizes by Horton orders $1-5$. Note that all distributions have a similar shape along the $y$-axis but are separated along the $x$-axis. (b) By rescaling each probability distribution by its respective mean collapses all the data onto a single curve. These data collapse demonstrates the striking self-similarity of the scaling relations within and across these populations.

Table 2. Branching ratios and mean family sizes for all data and by continent.

\begin{tabular}{lclllllll}
\hline sample & $\begin{array}{l}\text { sample size } \\
(n)\end{array}$ & $\begin{array}{l}\text { branching } \\
\text { ratio }(\bar{B})\end{array}$ & $\begin{array}{l}\text { 95\% CLs } \\
\text { (lower) }\end{array}$ & $\begin{array}{l}95 \% \text { CLs } \\
\text { (upper) }\end{array}$ & $\begin{array}{l}\text { sample size family size } \\
(n)\end{array}$ & $\begin{array}{l}\text { 95\% CLs } \\
(\bar{F})\end{array}$ & $\begin{array}{l}95 \% \text { CLs } \\
\text { (lower) }\end{array}$ \\
\hline all data & 339 & 3.77 & 3.68 & 3.86 & 114 & 4.48 & 4.31 & 4.67 \\
Asia & 29 & 3.37 & 3.10 & 3.71 & 17 & 3.89 & 3.67 & 4.11 \\
Africa & 19 & 3.46 & 2.81 & 3.67 & 6 & 4.53 & 4.22 & 4.84 \\
North America & 216 & 3.95 & 3.81 & 4.07 & 59 & 4.26 & 4.06 & 4.46 \\
Australia & 56 & 3.49 & 3.18 & 3.61 & 20 & 6.26 & 5.87 & 6.63 \\
South America & 19 & 3.51 & 2.70 & 3.92 & 12 & 4.52 & 4.03 & 5.12 \\
\hline
\end{tabular}

relations, we think this is unlikely. It is much more probable, as many ethnographers have emphasized that the family unit is the fundamental social unit in nearly all societies, and that families self-organize into a nested hierarchy of higher-level groups that are recognized both by the people themselves and by their ethnographers.

The hierarchical fractal-like organization of huntergatherer social systems is similar to the self-organized structures of other complex systems in nature (Arenas et al. 2001, 2004; Oltvai \& Barabasi 2002; Sole \& Bascompte 2006). We suggest that these complex social systems have been shaped by similar optimization processes operating to maximize whole-system performance. In the present case, these human social systems are hypothesized to reflect optimized networks of flows of essential commodities: food, other material resources, genes and culturally transmitted information. Individual foragers should maximize fitness by participating in social networks of exchanges that optimize the flow of resources. However, in density-dependent populations, individuals face tradeoffs between resource availability and competition from conspecifics, leading to optimization principles 
acting to regulate interactions and therefore network organization. It is these density-dependent tradeoffs that lead to the complex hierarchical structures we report here.

Yet, how do we account quantitatively for the branching ratio? We offer the following hypothesis. Recall from equation (3.2) that the branching ratio is simply the ratio of the frequency of group sizes between successive levels, $B=N\left(g_{\omega}\right) / N\left(g_{\omega+1}\right)$. This can be rearranged to be $N\left(g_{\omega}\right)=N\left(g_{\omega+1}\right) B$ or more generally $N\left(g_{\omega}\right)=N\left(g_{\Omega}\right) B^{\Omega-\omega}$, and as $N\left(g_{\Omega}\right) \equiv 1$, we have

$N\left(g_{\omega}\right)=B^{Q-\omega}$.

Further, as $N\left(g_{1}\right)=$ population size, $g_{\Omega}$, we then have $g_{\Omega}=B^{\Omega-1}$ or $g_{\Omega}=B^{5}$ in this case as $\Omega=6$. From equation (5.1), it follows that the number of families in a population scales with the branching ratio as $N\left(g_{2}\right)=B^{\Omega-2}$. As $N\left(g_{2}\right)=g_{\Omega} / g_{2}$ (see $\S 3$ ), we can write the branching ratio as $B=\left(g_{\Omega} / g_{2}\right)^{1 / \Omega-2}$ where family size, $g_{2}$, can be expressed in terms of the net reproductive rate, $R$, thus $g_{2}=2(R+1)$. Substituting this expression into the preceding equation, we then have

$B=\left(\frac{g_{\Omega}}{2(R+1)}\right)^{(1 /(\Omega-2))}$,

and rearranging, the net reproductive rate is them

$R=\frac{1}{2}\left(\frac{g_{\Omega}}{B}\right)^{(1 /(\Omega-2))}-1$.

Hence, as population size, $g_{\Omega}$, approaches $B^{\Omega-1}$, the net reproductive rate goes to 1 (i.e. reproductive replacement rates) as equation (5.3) reduces to $R=B / 2-1=1$. Therefore, at replacement rates, independent of population size or the number of levels in the network, the branching ratio reaches an equilibrium of 4 and follows the replacement family size of 4 (two parents and two offspring). It follows that in growing populations where the net reproductive rate $R>1$, the branching ratio should be less than the mean family size, and family size will be greater than 4 . Our results show that the mean branching ratio in our sample is approximately 3.8 , suggesting that huntergatherer populations are, on average, growing, predicting that mean family size should be greater than 4 . Indeed, mean family size is significantly greater than $4, F=4.48$ (4.30-4.67), giving a mean net reproductive rate $R=1.28$ (1.15-1.33), and a mean population growth rate $r=0.011$ (0.007-0.015) or approximately $1 \%$, where $r=\ln R / \tau$, and $\tau$ is generation time, approximately 20 years for traditional human populations under natural fertility conditions (Walker et al. 2006). Further, this model may explain the observed differences in average branching ratios across continents (table 2); hunter-gatherer populations in different continents may be in different stages of population growth (or decline) due to continent specific environmental, historical and demographic circumstances. However, on average, across all continents, populations are slightly below, but near reproductive replacement rates, as would be expected for a large, global sample of density-dependent populations.

This model has two important implications: (i) the branching ratio is a function of density-dependent reproduction in hierarchically structured populations and (ii) family-based relationships ramify in a hierarchical self-similar fashion up through the network. The second point is consistent with extensive ethnographic observations, where individuals move through social networks as families rather than individuals per se, which fuse into residential groups of multiple families to exploit resources efficiently, and fission along similar family lines to avoid conflict and inter-personal tension (e.g. desert aborigines (Gould 1969), Mbuti (Turnbull 1965), San (Tanaka 1980, 1989)).

We extend these ideas by noting that group dynamics are governed by two basic kinds of forces: (i) cohesive forces that tend to draw and hold individuals together and (ii) disruptive forces that tend to pull individuals apart and to create barriers to exchanges between them (see Chagnon 1975). Cohesive forces in hunter-gatherer groups include kin selection due to genetic relatedness, sharing of non-genetic information and exchange of material resources. There are clear cohesive forces within families and wider kin relations, but there are also cohesive forces that extend to larger groups at higher levels of the societal hierarchy. These include exchange of marriage partners so as to avoid inbreeding, communication of information about social and environmental conditions, and exchange of material resources through trade and commerce. Disruptive or antagonistic forces include competition for material resources and for mates, interpersonal conflict and disease epidemics. The intensity of competition, the balance between mutualistic and antagonistic interactions, and the probability of disease outbreak all increase with increasing group size, with the result that individuals aggregate into successively larger groups with successively decreasing frequencies and only for specific purposes, such as exchange of marriage partners, trade in goods that are not available locally, and defence against or competitive aggression (e.g. warfare) towards other higher-level groups.

The ideas we present here are not dissimilar from the concept of scalar stress first proposed by Johnson (1982) where group fissioning occurs, and structure emerges in egalitarian societies as a mechanism to dissipate social tension and maximize information processing capacity. However, our analyses emphasize the fundamental interplay of life-history constraints, the resource constraints of foraging within complex ecologies and the densitydependent effects of competition for energy, materials and information in finite environments in the emergence of a complex social structure. Our results are also similar in principle to those of Zhou et al. (2005) but there are quantitative differences. Indeed, we do find group size scaling relations in hunter-gatherer societies, but at a ratio of near 4, substantially greater than the ratio of near 3 reported in their study. However, these differences probably reflect the different sets of constraints operating on the different types of social network analysed in the respective studies. Given these differences, the qualitative similarities of discrete self-similar scaling ratios found in both studies are particularly interesting, adding support to Zhou et al.'s suggestion that such scaling ratios are a fundamental structural component of human social organization.

In hunter-gatherer societies all resources, including energy, materials and information, are transferred almost exclusively by direct human-to-human contact. It is then not surprising that the rates of flows diminish in a regular way from families to successively larger groups in accord with the 
effects of kinship and reciprocity on costs and benefits of exchanges among progressively larger groups with progressively reduced contacts. Of particular interest is the suggestion that genetic relatedness, exchanges of nongenetic information, and flows of material resources, may scale similarly throughout the social hierarchy. If true, selfsimilarity has profound implications for understanding the complex, interacting roles of genetic, ecological and social processes in the formation and maintenance of human societies on both shorter demographic and ecological scales, and longer evolutionary and biogeographic scales.

We thank Vijay Gupta, James Boone, Eric Charnov, Melanie Moses, Kim Hill, Ana Davidson, Robert Holdaway, Steve Litvin and the Brown lab for their valuable discussions and comments on earlier drafts. M.J.H., B.T.M., O.B. and J.H.B. gratefully acknowledge the support of NSF Biocomplexity grant DEB-0083422.

\section{REFERENCES}

Arenas, A., Díaz-Guilera, A. \& Guimerà, R. 2001 Communication in networks with hierarchical branching. Phys. Rev. Lett. 86, 3196-3199. (doi:10.1103/PhysRevLett.86.3196)

Arenas, A., Danon, L., Diaz-Guilera, A., Geiser, P. M. \& Guimera, R. 2004 Community analysis in social networks. Eur. Phys. F. B 38, 373-380. (doi:10.1140/epjb/e200400130-1)

Banavar, J. R., Maritan, A. \& Rinaldo, A. 1999 Size and form in efficient transportation networks. Nature 399, 130-132. (doi:10.1038/20144)

Banavar, J. R., Damuth, J., Maritan, A. \& Rinaldo, A. 2002 Supply-demand balance and metabolic scaling. Proc. Natl Acad. Sci. USA 99, 10 506-10 509. (doi:10.1073/pnas. 162216899)

Binford, L. R. 2001 Constructing frames of reference: an analytical method for archaeological theory building using ethnographic and environmental data sets. Berkeley, CA: University of California Press.

Birdsell, J. 1958 On population structure in generalized hunting and gathering populations. Evolution 12, 189-205. (doi:10.2307/2406029)

Birdsell, J. 1993 Microevolutionary patterns in aboriginal Australia. Oxford, UK: Oxford University Press.

Brown, J. H., Gupta, V. K., Li, B.-L., Milne, B. T., Restrepo, C. $\&$ West, G. B. 2002 The fractal nature of nature: power laws, ecological complexity and biodiversity. Phil. Trans. R. Soc. B 357, 619-626. (doi:10.1098/rstb.2001.0993)

Brown, J. H., Gillooly, J. F., Allen, A. P., Savage, V. M. \& West, G. B. 2004 Toward a metabolic theory of ecology. Ecology 85, 1771-1789. (doi:10.1890/03-9000)

Chagnon, N. A. 1975 Genealogy, solidarity, and relatedness: limits to local group size and patterns of fissioning in an expanding population. Yearb. Phys. Anthropol. 19, 95-110.

Colizza, V., Banavar, J. R., Maritan, A. \& Rinaldo, A. 2004 Network structures from selection principles. Phys. Rev. Lett. 92, 198 701. (doi:10.1103/PhysRevLett.92.198701)

Dunbar, R. I. M. 1993 Coevolution of neocortex size, group size and language in humans. Behav. Brain Sci. 16, 681-735.

Enquist, B. J., West, G. B. \& Brown, J. H. 2000 Quarterpower allometric scaling in vascular plants: functional basis and ecological consequences. In Scaling in biology (ed. G. B. West), p. 352. Oxford, UK: Oxford University Press.

Gamble, C. 1999 The paleolithic societies of Europe. Cambridge, UK: Cambridge University Press.

Gould, R. A. 1969 Yiwara: foragers of the Australian desert. New York, NY: Charles Scribner's and Sons.
Guimera, R., Danaon, L., Diaz-Guilera, A., Giralt, F. \& Arenas, A. 2003 Self-similar community structure in a network of human interactions. Phys. Rev. E 68, 065103. (doi:10.1103/PhysRevE.68.065103)

Gurven, M. 2004 To give and give not: the behavioral ecology of human food transfers. Behav. Brain Sci. 27, 543-559.

Gurven, M. \& Walker, R. 2006 Energetic demand of multiple dependents and the evolution of slow human growth. Proc. R. Soc. B 273, 835-841. (doi:10.1098/rspb.2005.3380)

Hamilton, M. J., Milne, B. T., Walker, R. S. \& Brown, J. H. 2007 Nonlinear scaling of space use in human huntergatherers. Proc. Natl Acad. Sci. USA 104, 4765-4769. (doi:10.1073/pnas.0611197104)

Johnson, G. A. 1982 Organizational structure and scalar stress. In Theory and explanation in archaeology (eds C. Renfrew, M. Rowlands \& B. A. Seagraves), pp. 389-421. New York, NY: Academic Press.

Kaplan, H., Hill, K., Lancaster, J. \& Hurtado, A. M. 2000 A theory of human life history evolution: diet, intelligence, and longevity. Evol. Anthropol. 9, 156-185. (doi:10.1002/ 1520-6505(2000)9:4<156::AID-EVAN5>3.0.CO;2-7)

Kelly, R. L. 1995 The foraging spectrum: diversity in huntergatherer lifeways. Washington, DC: Smithsonian Institution Press.

Lourandos, H. 1997 Continent of hunter-gatherers: new perspectives in Australian prehistory. Cambridge, UK: Cambridge University Press.

Maschner, H. D. G. \& Bentley, R. A. 2003 The power law of rank and household on the North Pacific. In Complex systems and archaeology (eds R. A. Bentley \& H. D.-G. Maschner), pp. 47-60. Salt Lake City, UT: University of Utah Press.

Newman, M. E. J. 2003 The structure and function of complex networks. SIAM Rev. 45, 167-256. (doi:10. $1137 / \mathrm{S} 003614450342480$ )

Newman, M. E. J. 2004 Coauthorship networks and patterns of scientific collaboration. Proc. Natl Acad. Sci. USA 101, 5200-5205. (doi:10.1073/pnas.0307545100)

Oltvai, Z. N. \& Barabasi, A.-L. 2002 Life's complexity pyramid. Science 298, 763-764. (doi:10.1126/science. 1078563)

Peckham, S. \& Gupta, V. K. 1999 A reformulation of Horton laws for large river networks in terms of statistical selfsimilarity. Water Resour. Res. 35, 2763-2777. (doi:10. 1029/1999WR900154)

Porter, M. A., Mucha, P. J., Newman, M. E. J. \& Warmbrand, C. M. 2005 A network analysis of committees in the U.S. House of representatives. Proc. Natl Acad. Sci. USA 102, 7057-7062. (doi:10.1073/pnas.0500191102)

Ravasz, E. \& Barabási, A.-L. 2003 Hierarchical organization in complex networks. Phys. Rev. E 67, 026112. (doi:10. 1103/PhysRevE.67.026112)

Rinaldo, A., Banavar, J. R. \& Maritan, A. 2006 Trees, networks, and hydrology. Water Resour. Res. 42, W06D07. (doi:10.1029/2005WR004108)

Rodriguez-Iturbe, I. \& Rinaldo, A. 1997 Fractal river basins: chance and self-organization. Cambridge, UK: Cambridge University Press.

Schneider, E. D. \& Kay, J. K. 1994 Life as a manifestation of the second law of thermodynamics. Math. Comput. Modell. 19, 25-48. (doi:10.1016/0895-7177(94)90188-0)

Sole, R. V. \& Bascompte, J. 2006 Self-organization in complex ecosystems. Princeton, NJ: Princeton University Press.

Stewart, J. H. 1938 Basin-plateau aboriginal sociopolitical groups. Smithsonian Institution Bureau of American Ethnology Bulletin. Salt Lake City, UT: University of Utah Press. 
Tanaka, J. 1980 The San hunter-gatherers of the Kalahari: a study in ecological anthropology. Tokyo, Japan: University of Tokyo Press.

Tanaka, J. 1989 Social integration of the San society from the viewpoint of sexual relationships. Afr. Study Monogr. 9, 153-165.

Turnbull, C. M. 1965 Wayward servants: the two Worlds of the African Pygmies. Garden City, NY: Natural History Press.

Veitzer, S. \& Gupta, V. K. 2000 Random self-similar river networks and derivations of generalized Horton laws in terms of statistical simple scaling. Water Resour. Res. 36, 1033-1048. (doi:10.1029/1999WR900327)

Walker, R. S. et al. 2006 Growth rates and life histories in twenty-two small-scale societies. Am. F. Hum. Biol. 18, 295-311. (doi:10.1002/ajhb.20510)

West, G. B. \& Brown, J. H. 2005 The origin of allometric scaling laws in biology from genomes to ecosystems: towards a quantitative unifying theory of biological structure and organization. F. Exp. Biol. 208, 1575-1592. (doi:10.1242/jeb.01589)

West, G. B., Brown, J. H. \& Enquist, B. J. 1997 A general model for the origin of allometric scaling laws in biology. Science 276, 122-126. (doi:10.1126/science.276. 5309.122)

West, G. B., Brown, J. H. \& Enquist, B. J. 1999 The fourth dimension of life: fractal geometry and allometric scaling of organisms. Science 284, 1677-1679. (doi:10.1126/ science.284.5420.1677)

Wobst, H. M. 1974 Boundary conditions for paleolithic social systems: a simulation approach. Am. Antiq. 39, 147-178. (doi:10.2307/279579)

Zhou, W., Sornette, D., Hill, R. \& Dunbar, R. 2005 Discrete hierarchical organization of social group sizes. Proc. $R$. Soc. B 272, 439-444. (doi:10.1098/rspb.2004.2970) 


\section{NOTICE OF CORRECTION}

Figure 1 and paragraph 3 of page 2 are now presented in the correct form.

10 July 2007 